\title{
Delayed catastrophic thrombotic events in young and asymptomatic post COVID-19 patients
}

\author{
Bingwen Eugene Fan ${ }^{1,2,3,4}$ (1) Thirugnanam Umapathi ${ }^{3,4,5} \cdot$ Karen Chua $^{3,4,6} \cdot$ Yew Woon Chia ${ }^{3,4,7}$. \\ Shiun Woei Wong ${ }^{3,4,7}$. Glenn Wei Leong $\operatorname{Tan}^{3,4,8}$. Sadhana Chandrasekar ${ }^{3,4,8} \cdot$ Ying Hui Lum $^{6}$. Shawn Vasoo ${ }^{3,4,9,10}$. \\ Rinkoo Dalan ${ }^{3,4,11}$
}

Accepted: 31 October 2020 / Published online: 7 November 2020

(c) Springer Science+Business Media, LLC, part of Springer Nature 2020

We read with interest the editorial "Anticipating the longterm cardiovascular effects of COVID-19 [1]" which featured COVID-19 end-organ thrombotic complications. COVID-19 associated hypercoagulability [2] and increased thromboembolism in COVID-19 has been well described [3]. While the editorial raised the key question about "potential long-term cardiovascular effects (of COVID-19)", little is known about the post COVID-19 vascular complications. In response, we would like to highlight a series of catastrophic arterial events observed in post COVID-19 patients (Table 1), echoing the Editor's concerns of post-infectious vasculopathy.

Bingwen Eugene Fan

Bingwen_Eugene_Fan@ttsh.com.sg

1 Department of Haematology, Tan Tock Seng Hospital, Singapore, Singapore

2 Department of Laboratory Medicine, Khoo Teck Puat Hospital, Singapore, Singapore

3 Lee Kong Chian School of Medicine, Singapore, Singapore

4 Yong Loo Lin School of Medicine, Singapore, Singapore

5 Department of Neurology, National Neuroscience Institute (Tan Tock Seng Hospital Campus), Singapore, Singapore

6 Department of Rehabilitation Medicine, Tan Tock Seng Hospital Rehabilitation Centre, Singapore, Singapore

7 Department of Cardiology, Tan Tock Seng Hospital, Singapore, Singapore

8 Department of General Surgery, Tan Tock Seng Hospital, Singapore, Singapore

9 Department of Infectious Diseases, Tan Tock Seng Hospital, Singapore, Singapore

10 National Centre for Infectious Diseases, Singapore, Singapore

11 Department of Endocrinology, Tan Tock Seng Hospital, Singapore, Singapore
During the months of April 2020 to July 2020, Singapore experienced a surge in COVID-19 cases amongst our migrant workers. Subsequently, in July 2020 to September 2020, 4 young healthy migrant workers presented with catastrophic, large arterial thromboses. They were detected to have COVID-19 during routine screening of affected migrant workers' dormitories. They had raised Immunoglobulin G for SARS-CoV-2 (Roche Elecsys Anti-SARSCoV-2) indicative of COVID-19 seroconversion. They were asymptomatic and did not meet criteria necessitating hospitalization nor thromboprophylaxis and were quarantined at isolation facilities. Subsequently, they presented with a sentinel thrombotic event at a median of 78 days from seroconversion. Their median age was 38.5 years, and they were of South Asian ethnicity. Cardiovascular risk factors screening showed that one had recently diagnosed diabetes mellitus with a HbA1c of $6.7 \%$ whilst the others did not have any risk factors.

Two patients suffered from severe large vessel ischaemic strokes (Fig. 1a-d), one had an acute ischaemic limb (Fig. 2a) due to emboli from an aortic thrombosis (Fig. 2b), and one had anterolateral ST-Elevation Myocardial Infarction (Fig. 3a-c). No features of overt arteriosclerotic disease were found during neuroimaging, CT angiography and coronary angiography. Thrombophilia screen and stroke evaluation inclusive of carotid imaging, echocardiography, 24-h holter monitoring were normal.

The remarkable features of these cases include young age without preexisting cardiovascular risk factors, asymptomatic SARS-CoV-2 infection and the long latency between initial positive serology and their catastrophic vascular event. Firstly, two patients had an unusual site of thrombosis, with mobile aortic thromboses discovered on CT imaging. Aortic thrombosis is rare in young patients without a significant thromboembolic risk factors or pre-existing arteriosclerosis. The SARS-CoV-2 virus is 


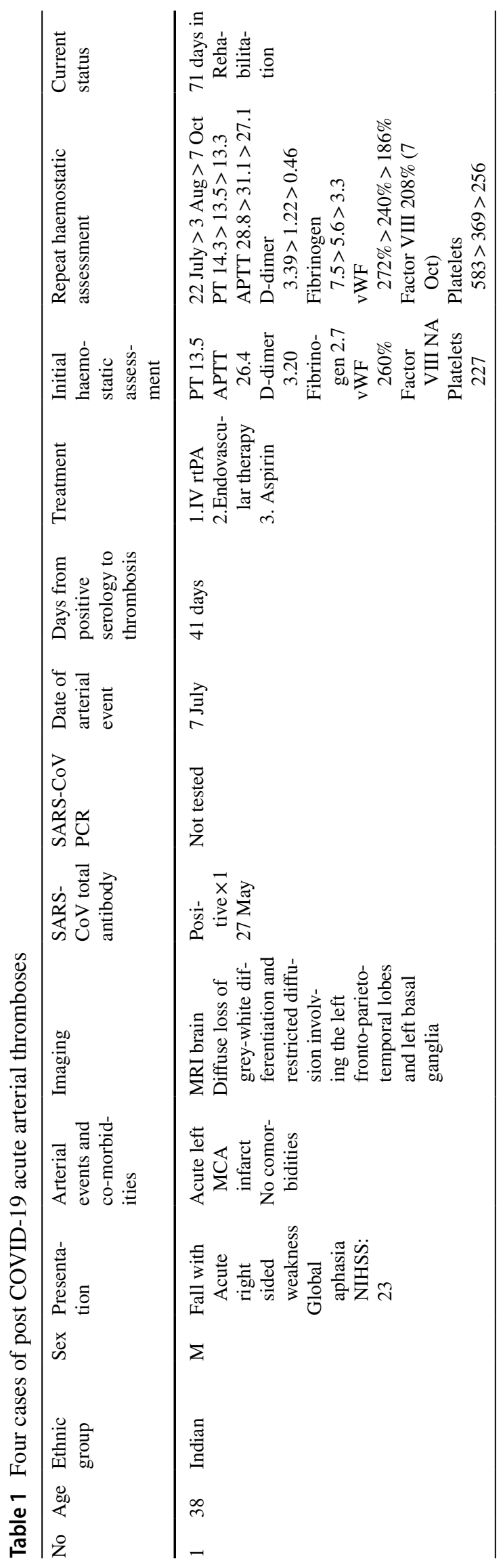




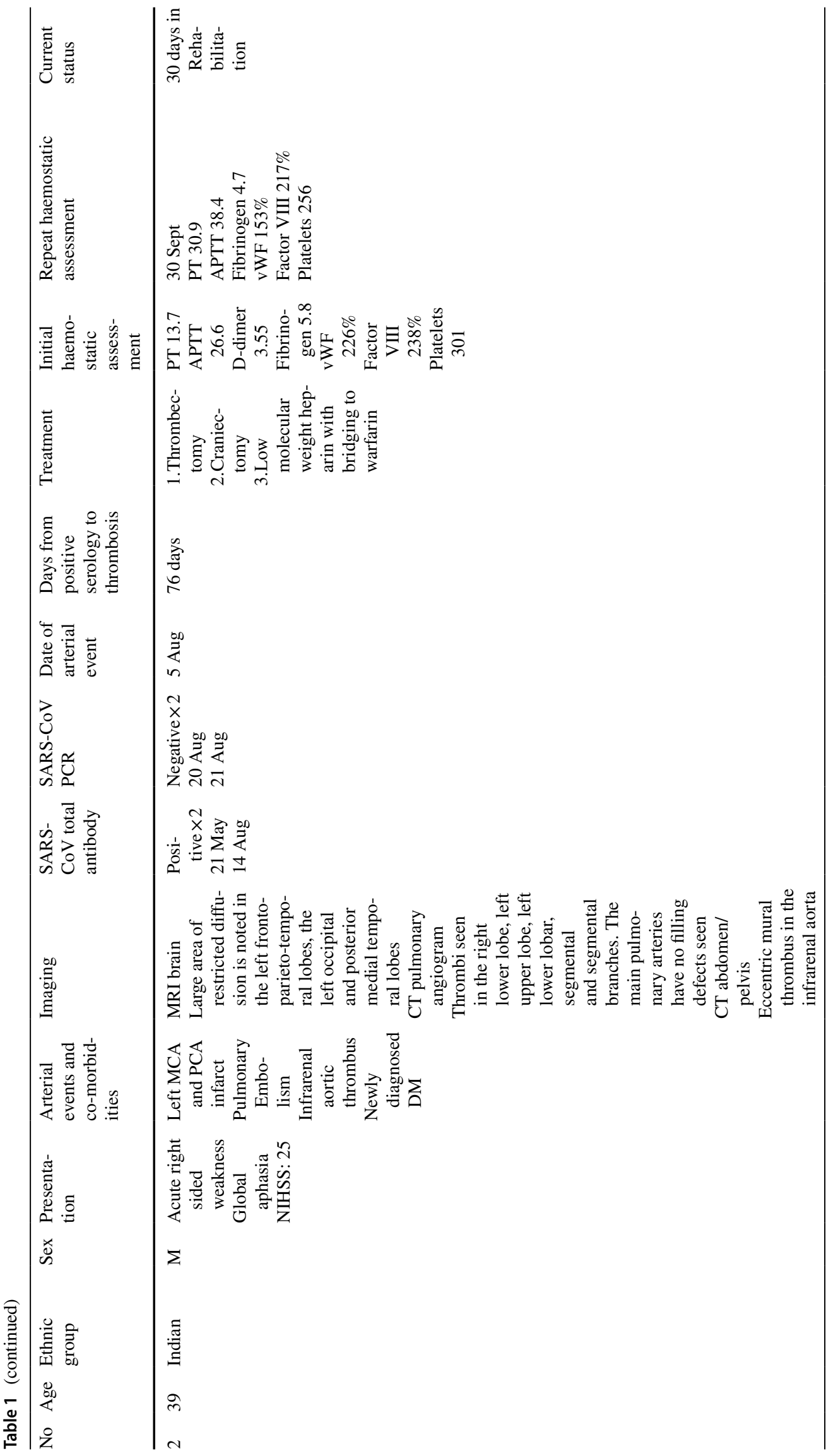




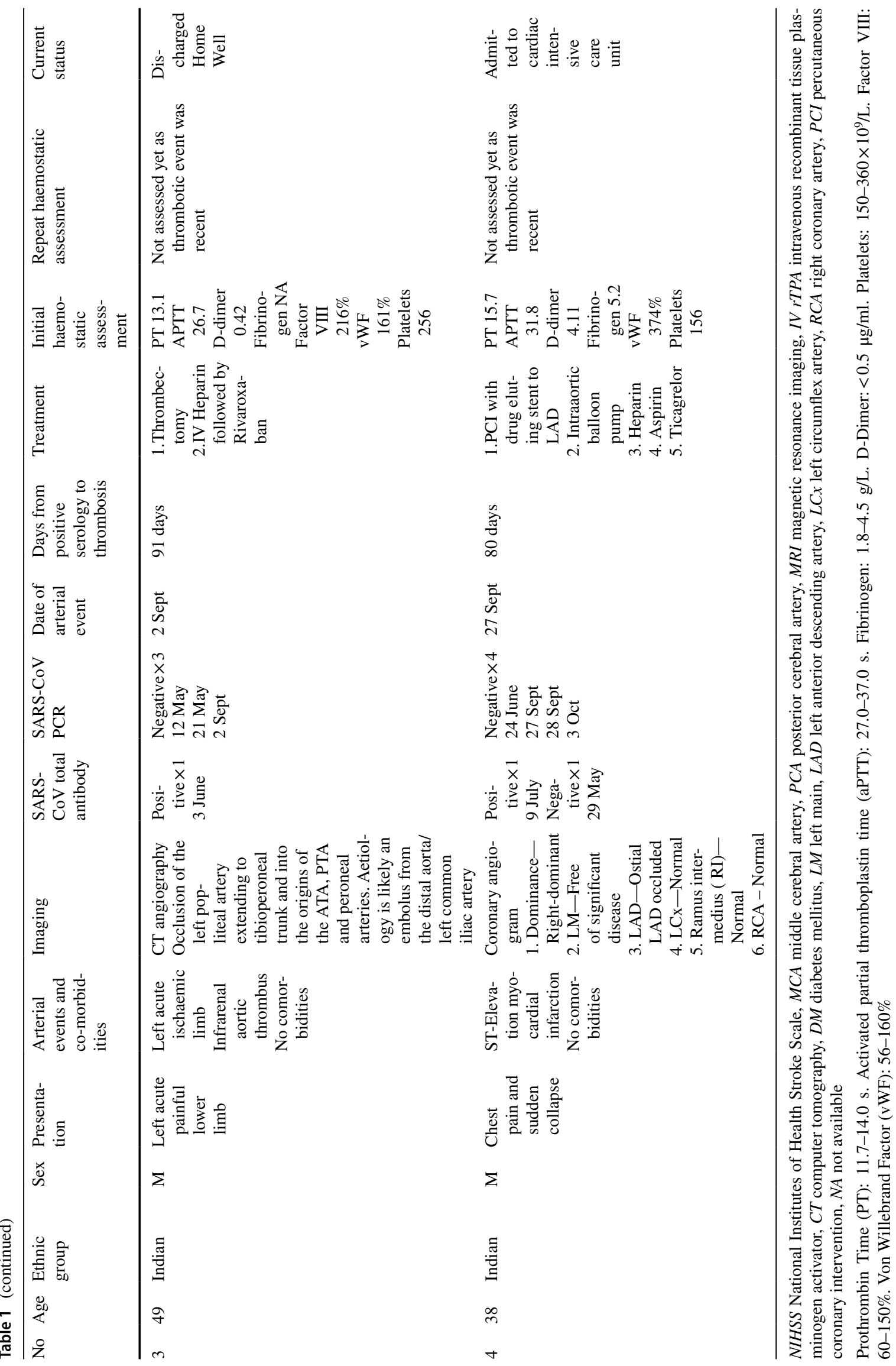


Fig. 1 a-d Acute magnetic resonance brain images of the 2 patients with large vessel ischaemic stroke. They show large areas of restricted diffusion in left middle cerebral, and middle cerebral, posterior cerebral artery territories, respectively. Adjoining intracranial magnetic resonance angiograms show loss of normal flow void in the left internal carotid and middle cerebral arteries in the first patient $(\mathbf{a}, \mathbf{b})$, and $\mathrm{M} 1$ segment of left middle cerebral artery in the second patient $(\mathbf{c}, \mathbf{d})$

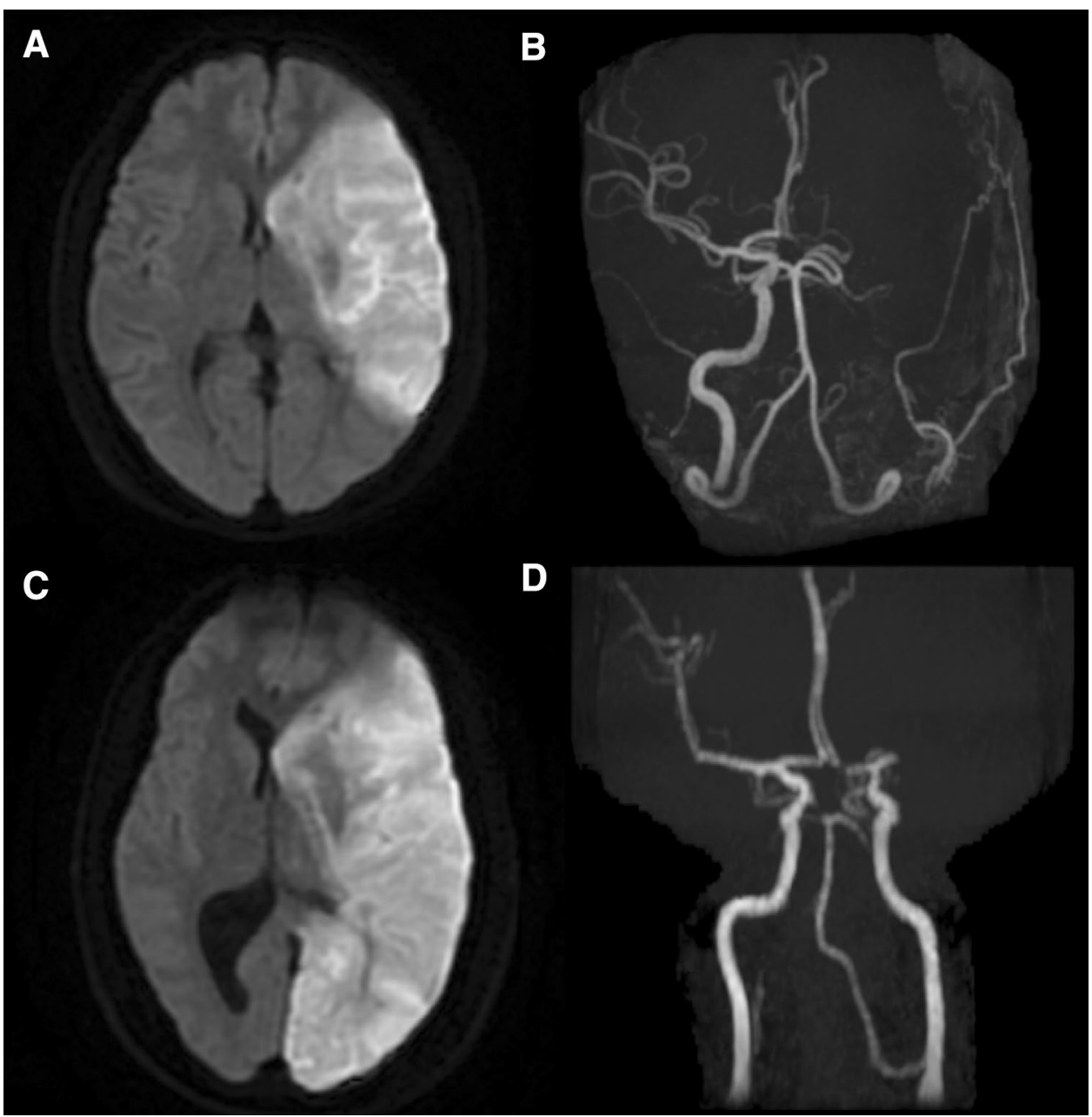

known to cause endothelial damage during acute infection $[4,5]$ and cases of COVID-19 associated acute ischaemic limb secondary to embolism from aortic thrombosis have been described [6, 7]. However, less is known about persistence of endotheliitis in the convalescent phase of illness. We postulate that post SARS-CoV-2 infection, a state of endothelial activation with downstream signaling pathways of low-grade inflammation and thrombosis may persist [8]. Similar mural thrombosis has been described in patients on chemotherapy and has been attributed to trauma to vascular endothelium and a hypercoagulable state [9]. However, our patients did not consume any medications and did not have any endovascular lines. The aortic thrombosis in COVID-19 could be a consequence of persistent endothelial dysfunction due to viral inclusion bodies at specific sites (this has been reported in Chilblains ('COVID toes') in children and young adults [10]) Secondly, haemostatic assessment of the 4 patients at time of acute thrombosis highly suggested a hypercoagulable state, with a markedly raised Factor VIII, elevated von Willebrand factor antigen, increased D-dimer levels and hyperfibrinogenaemia. Both endothelial dysfunction and hypercoagulability are 2 key factors in Virchow's triad for thrombosis. The post-thrombosis evaluation of the haemostatic profile in 2 of our patients with ischaemic stroke at a 2 to 3-month window from onset of acute stroke revealed declining but persistently raised von Willebrand factor antigen, Factor VIII, and fibrinogen levels. A persistently raised von Willebrand factor may reflect chronic endothelial activation and dysfunction [11], as well as ongoing platelet hyperactivation [12]. Thirdly, for the young patient with cardiac arrest and acute myocardial infarction, COVID-19 may have induced an 'accelerated atherosclerosis' and precipitated a coronary event during his convalescence. There was complete occlusion of left anterior descending (LAD) artery (Fig. 3a). This is suggested by findings from Intravascular ultrasound (Volcano Eagle Eye catheter) which was used to interrogate the LAD artery. Small amount of clot was retrieved using thrombectomy device. It showed a focal heavily fibrous plaque burden of $59 \%$ and minimal lumen diameter of $2.1 \mathrm{~mm}$ in the ostial LAD (Fig. 3c), with a plaque burden of more than $70 \%$ and 

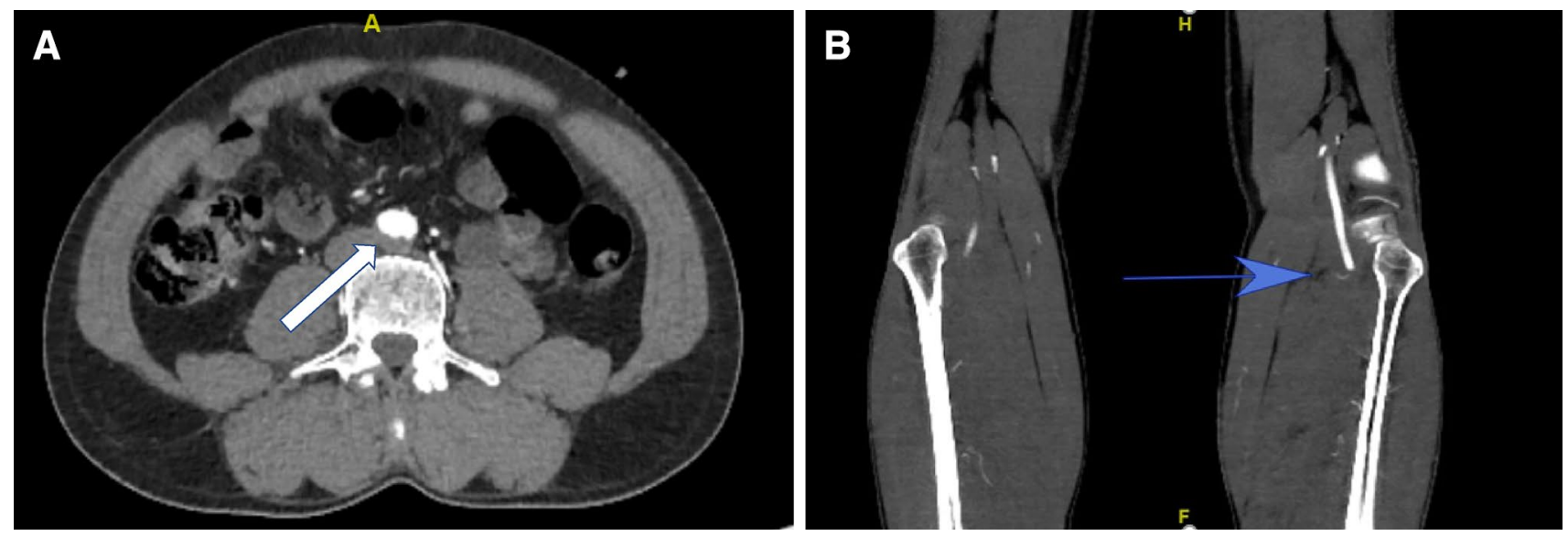

Fig. 2 a An eccentric thrombus (white arrow) is present in the distal aorta just before its bifurcation. b An abrupt cut off of the left popliteal artery (blue arrow)

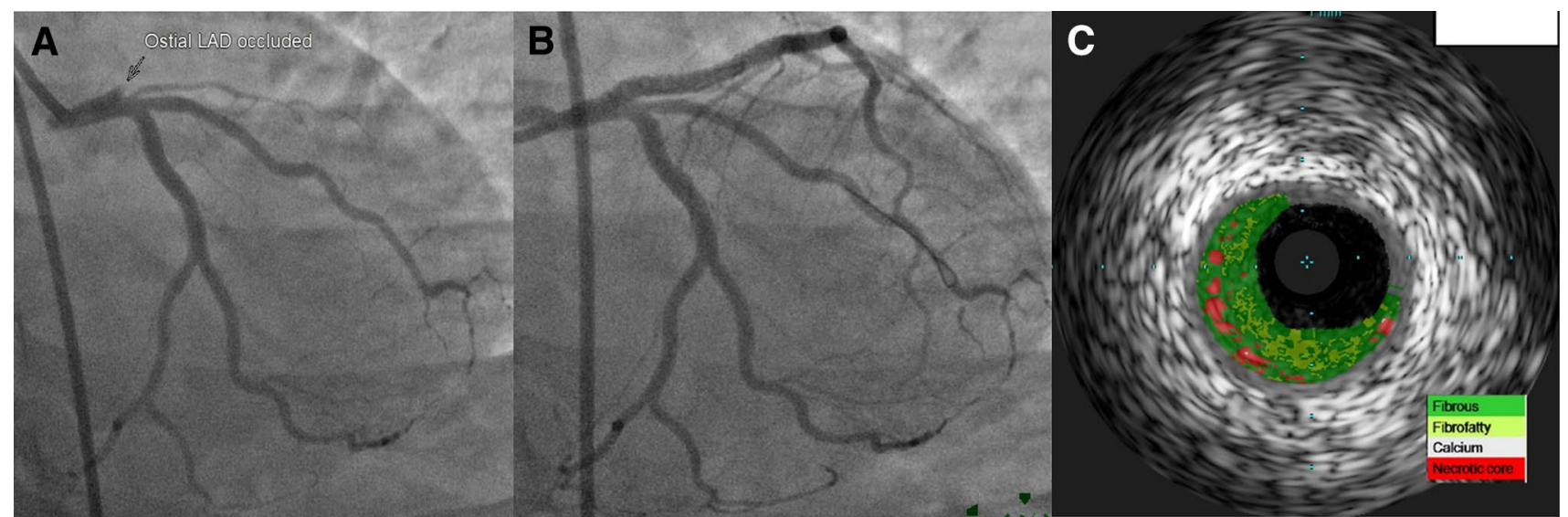

Fig. 3 a Coronary angiogram showed complete occlusion of ostial left anterior descending artery (LAD). Left circumflex artery and ramus intermedius branch are normal. b Restoration of flow in the

minimal lumen area of less than $4 \mathrm{~mm}^{2}$ being independent predictors of major adverse cardiovascular events [13]. This is unusual as fibrotic lesions are usually deprived from lipid and inflammatory cells and hence less likely to rupture and generate thrombosis [14].

COVID-19 is known to cause cardiac complications with a case series from China reporting a high percentage (19.7\%) of COVID 19 patients having evidence of myocardial injury with $51 \%$ in-hospital mortality compared with $4.5 \%$ without raised troponin [15]. Potential mechanisms that explain cardiac injury during acute infection include (i) imbalance in myocardial demand and supply due to tachycardia and hypotension from type 2 myocardial infarction; (ii) acute atherothrombosis in a virally induced thrombotic and inflammatory milieu; (iii) microvascular dysfunction from microthrombi or endothelial injury; (iv) stress-related
LAD after implantation of drug eluting stent. c Intravascular ultrasound (virtual histology) showed predominantly fibrotic plaque with minimal necrotic core in the ostial LAD

cardiomyopathy (Takotsubo) and (v) direct cardiomyocyte toxicity and myocarditis, of which artherothrombosis as well as direct cardiomyocyte toxicity may cause sustained longterm damage to the coronary vessels and the myocardium, with 10 patients in Germany critically ill with COVID-19 demonstrating low to no detectable virus in myocardial tissues despite multiple microemboli in the heart [16]. These observations suggest that the SARS-CoV-2 virus poses significant and chronic immuno-thrombogenicity which may cumulate eventually in a major thrombotic event.

Although small, our case series suggests that catastrophic vascular events can occur unexpectedly in fit patients with mild or asymptomatic COVID-19 infection and may unpredictably happen many weeks later.

The COVID-19 pandemic has a prolonged and serious impact on the cardiovascular health of patients. Hence for 
convalescent patients, regardless of the severity of infection and the absence of co-morbidities, clinicians should remain vigilant for post-infective thrombotic sequelae as well as consider screening for and closely managing cardiovascular risk factors. Multi-disciplinary collaboration into the epidemiology, pathogenesis and treatment of COVID-19 associated thrombosis and in evaluating the utility of extended thromboprophylaxis for COVID-19 patients should be strongly considered.

Author contributions BEF and RD conceived the manuscript. All authors made substantial contributions in analysing the data, revising it critically for important intellectual content and editing the manuscript.

Funding No funding for this study was obtained. All authors had full access to the full data in the study and accept responsibility to submit for publication.

\section{Compliance with ethical standards}

Conflict of interest The authors declare that they have no conflict of interest.

Ethical approval A waiver of informed consent was provided by the Ministry of Health (Singapore), under the provisions of the Infectious Diseases Act.

\section{References}

1. Becker RC (2020a) Anticipating the long-term cardiovascular effects of COVID-19. J Thromb Thrombolysis 50:512-524

2. Fan BE, Ng J, Chan SSW et al (2020) COVID-19 associated coagulopathy in critically ill patients: a hypercoagulable state demonstrated by parameters of haemostasis and clot waveform analysis. J Thromb Thrombolysis. https://doi.org/10.1007/s1123 9-020-02318-x

3. Klok FA, Kruip MJHA, van der Meer NJM, Arbous MS, Gommers DAMPJ, Kant KM, Kaptein FHJ, van Paassen J, Stals MAM, Huisman MV, Endeman H (2020) Incidence of thrombotic complications in critically ill ICU patients with COVID-19. Thromb Res 191:145-147

4. Varga Z, Flammer AJ, Steiger P et al (2020) Endothelial cell infection and endotheliitis in COVID-19. Lancet 395(10234):1417-1418
5. Becker RC (2020b) COVID-19-associated vasculitis and vasculopathy. J Thromb Thrombolysis 50(3):499-511. https://doi. org/10.1007/s11239-020-02230-4

6. Goh SSN, Yong EM, Hong QT et al (2020) Acute aortic thrombosis presenting as acute limb ischemia in two young, non-atherosclerotic patients. Br J Surg 107:e565-e566

7. Fan BE, Chia YW, Sum CLL et al (2020) Global haemostatic tests in rapid diagnosis and management of COVID-19 associated coagulopathy in acute limb ischaemia. J Thromb Thrombolysis 50(2):292-297. https://doi.org/10.1007/s11239-020-02165-w

8. Dalan R, Boehm BO (2020) The implications of COVID-19 infection on the endothelium: a metabolic vascular perspective. Diabetes Metab Res Rev. https://doi.org/10.1002/dmrr.3402

9. Starling N, Rao S, Cunningham D, Iveson T et al (2009) Thromboembolism in patients with advanced gastroesophageal cancer treated with anthracycline, platinum, and fluoropyrimidine combination chemotherapy: a report from the UK National Cancer Research Institute Upper Gastrointestinal Clinical Studies Group. J Clin Oncol 10:3786-3793

10. Colmenero I, Santonja C, Alonso-Riaño M et al (2020) SARSCoV-2 endothelial infection causes COVID-19 chilblains: histopathological, immunohistochemical and ultrastructural study of seven paediatric cases. Br J Dermatol 183:729-737

11. Goshua G, Pine AB, Meizlish ML et al (2020) Endotheliopathy in COVID-19-associated coagulopathy: evidence from a singlecentre, cross-sectional study. Lancet Haematol 7:e575-e582

12. Zaid Y, Puhm F, Allaeys I et al (2020) Platelets can associate with SARS-Cov-2 RNA and are hyperactivated in COVID-19. Circ Res. https://doi.org/10.1161/CIRCRESAHA.120.317703

13. Moreno PR, Narula J (2014) Thinking outside the lumen: fractional flow reserve versus intravascular imaging for major adverse cardiac event prediction. J Am Coll Cardiol 63:1141-1144

14. Bharadwaj AS, Vengrenyuk Y, Yoshimura T, Baber U, Hasan C et al (2016) Multimodality intravascular imaging to evaluate sex difference in plaque morphology in stable CAD. J Am Coll Cardiol 9(4):400-407

15. Shi S, Qin M, Shen B et al (2020) Association of cardiac injury with mortality in hospitalized patients with COVID-19 in Wuhan China. JAMA Cardiol 5(7):802-810

16. Guagliumi G, Sonzogni A, Pescetelli I, Pellegrini D, Finn AV (2020) Microthrombic and ST-segment elevation myocardial infarction in COVID-10. Circulation 142(8):804-809

Publisher's Note Springer Nature remains neutral with regard to jurisdictional claims in published maps and institutional affiliations. 